\title{
Anterior Interosseous Nerve Transfer in Ulnar Nerve Injury
}

S.M.Monib,W.A.Kandil,O.M.Essawy and S.M.Zahid

Orthopedic Dept.,Faculty of Medicine, Benha Univ., Benha, Egypt

E-Mail:samir@gmail.com

\begin{abstract}
Wounds to the ulnar nerve bring about both tactile and engine shortfalls inside the hand. This examination planned to assess practical result of move foremost interossous nerve to profound engine part of ulnar nerve injury. also, to contrast our outcome and different focuses result doing likewise tequnique or other tequniques. Ten patients, 7 guys and 3 females with a mean time of $28.5 \pm 6.86$ with least 18 and most extreme 42 years of age were remembered for this investigation. The interim span before a medical procedure was $4.25 \pm 1.48$ with least 2 months and most extreme a half year. All patients experienced proximal high ulnar nerve injury and were incorporated for distal neurotization. All patients had been submitted to 1ry fix of ulnar nerve at season of injury. there was huge connection between better engine result and more limited time span before a medical procedure. Likewise there was a critical connection between better engine result and consistence of the patients to physical thrapy. The useful misfortune was missing after denervation of pronator quadrates as the pronator teres can repay in all the examined patients. Hold and squeeze strength were improved in all patients to an agreeable level. From the hand specialist's perspective the outcomes are empowering in overseeing such cases with the nerve move method to reestablish capacity to natural muscles of hand.
\end{abstract}

Keyword: Interosseous, Nerve, Ulnar, Injury.

\section{Introduction}

Wounds to the ulnar nerve bring about both tactile and engine shortfalls inside the hand. Ulnar nerve wounds can be generally separated into low wounds and high wounds. In low ulnar nerve wounds, the nerve is harmed distal to the engine part of the Flexor carpi ulnaris (FCU) and engine branch to the Flexor digitorum profundus (FDP) of the ring and little fingers. In low wounds, reasonableness to the palmar ulnar hand is lost and loss of motion happens for the most part to each of the 7 interossei, the ulnar 2 lumbrical, the 3 hypothenar, the adductor pollicis, and the profound top of the flexor pollicis brevis muscles [1].

Significant level nerve wounds lead to the deficiency of both hold and squeeze strength in the hand, and sensation in the little finger and the ulnar side of the ring finger. In high wounds, the nerve is harmed over the source of the engine part of the FCU and FDP muscles [2].

The deficiency of inborn muscle work brings about a powerlessness to flex at the metacarpo-phalangeal (MCP) joints and expand the interphalan-geal (IP) joints. This result;s in the improvement of the characteristic less or hook stance of the ring and little fingers, where there is hyperex-pressure at the MCP joints and flexion at the IP joints (Duchenne's sign). Axonal recovery advances after fringe nerve sore is at a pace of 1-2 mm/day. Since muscle filaments go through irreversible changes following a year of denervation, it is basic that treat-ment be embraced immediately for practical recuperation [3].

Proximal sores in the arm, in any event, when treated inside 3 months following injury, convey a high danger of causing irreversible muscle decay before the recovering axons can arrive at the engine end plates. The greater part of the arrangement detailed just $20 \%$ of M4 or M5 when the re-pair is acted in a situation around the degree of the elbow, irrespec-tive of the utilization of unions. Because of these restricted outcomes got with the nerve fix, it has been suggested that distal ligament moves ought to be offered as the best option careful intercession for such cas-es, debilitating the nerve a medical procedure. Nonetheless, Tendon exchanges in the present circumstance are viewed as auxiliary rescue strategies and have been found to give just restricted outcomes [4].

Ligament move might be demonstrated to address ripping at and frail thumb-forefinger squeeze and for rectification of feeble FDP flexion at the ring and little fingers. Nonetheless, it is a long ways past in capacity than miniature neuro careful fix. Methods have been intended to forestall natural decay dur-ing proximal nerve recuperation. portray the utilization of interpositional nerve unites set distally between the ulnar and the middle engine branches [5].

Nerve moves depend on the hypothesis that it changes over the prox-imal nerve injury into a distal nerve injury by moving a unim-portant nerve to the more basic or significant nerve. Nerve move portrayed as move of Anterior interosseus nerve (AIN) to profound part of ulnar nerve to reestablish engine work either unique start to finish or all the more as of late "supercharged finish to-side nerve moves" (SETS) as named by Mackinnon and partners when pri-may fix has been done [4].

The aftereffects of start to finish move give some improvement to forestall ripping at, improve squeeze strength, and deter the requirement for ligament moves. Nonetheless, in this regular nerve move, the objective organs are completely innervated by the moved nerve and not by the ulnar nerve so the first nerve reinnervation is aban-doned completely. These variables lead to deficient practical recuperation where the best outcome acquire was not more than grade 3 recuperation of muscle strength by clinical exploration board scale [6].

Ongoing examinations have shown that nerve move should be possible in finish to side style where the giver nerve could grow through the epineurial window of the harmed beneficiary nerve, build up axon end plate 
associations at early time and doubly innervate target organs by both a benefactor and unique nerve [7].

This investigation expected to assess utilitarian result of move foremost interossous nerve to profound engine part of ulnar nerve injury. What's more, to Compare Our Result With Other Centers result Doing The Same Tequnique or Other Tequniques.

\section{Patients and methods}

This study has been conducted at the orthopedic department, faculty of medicine, Benha university hospitals in the period from $12 / 2018$ to $2 / 2020$. Ten patients, 7 males and 3 females with high ulnar nerve injury.

\section{Inclusion criteria}

To be included in the study, the patient must fulfill all the following criteria:

- Site of the injury: at or above level of elbow and proximal to FCU branch origin $(10 \mathrm{~cm}$ or more to proximal wrist crease).

- Nature of the injury; post traumatic injury.

- Sex; both males and females.

- Age; any age.

\section{Exclusion criteria}

The patients with any of the following criteria were excluded from the study:

- Any patient with non-post traumatic injury either inflammatory or tumor caused palsy or idiopathic.

- Any distal injury below origin of FCU branch.
- Any other concomitant distal injury of the nerve in the wrist or fractures.

- Delayed presentation after 6 months of injury.

\section{Preoperative management and evaluation}

A full history was taken including age, sex, cause of the lesion and relevant general health problems especially diabetes, hypertension, and smoking.

Evaluation of the affected limb:

Examination of the intrinsic muscles of affected limb was done to all patients as follows:

- Examination by card test to test adductor pollicis muscle. Froment's sign was found + in all patients.

- Examination of abduction to test palmar inter-osseii. No active range of motion and no palpable muscle contraction was found in all patients.

- Examination of adduction of fingers to test dorsal inter-osseii. No active range of motion and no palpable muscle contraction was found in all patients.

- Examination of flexion of MCP joint to test lumbricals. No active range of motion and no palpable muscle contraction was found in all patients.

- Examination of sensation for medial $1 \& 1 / 2$ fingers.

- Wasting in the hand (guttering on the dorsum of the hand due to atrophy of the interossie- wasting in the $1^{\text {st }}$ web space doe to the atrophy of the adductor pollicis wasting in the hypothenar eminence)

- Presence of specific deformity (partial claw hands hyperextension of the MP joint with flexion of the IP joints of the ulnar 2 fingers)
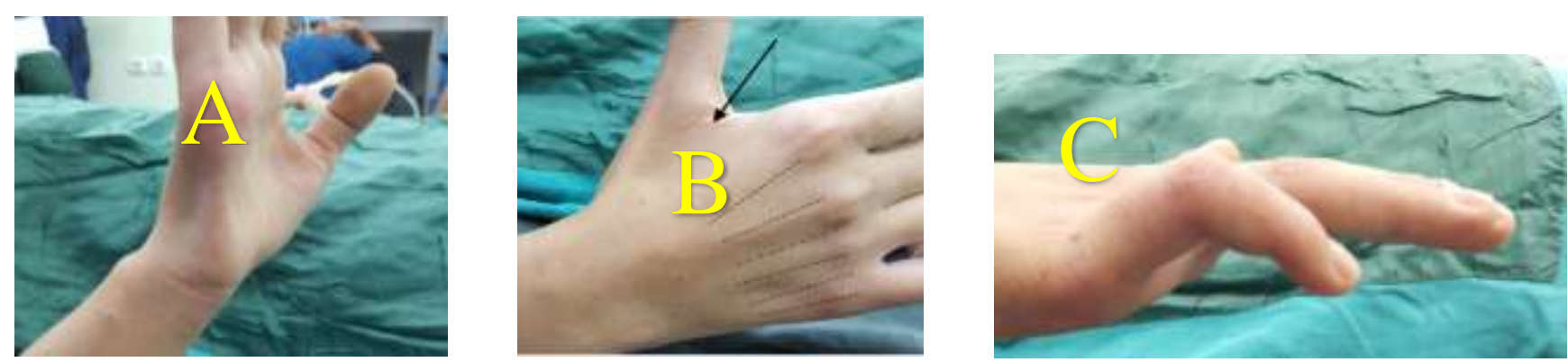

Fig.1a: Wasting in the1st web space and guttering b; Wasting in the hypothenar eminence c: Partial claw hand deformity

\section{ROM}

Active and passive ROM had to be measured in all patients and lag is detected and documented using a goniometer

Investigations:

\section{Radiological}

- Plain X-ray was done for all patients on the affected limb.

- EMG and nerve conduction velocity was done for the affected limb for ulnar nerve supplied muscles.
- Superficial U/S was done on ulnar nerve to ensure complete tear of nerve.

\section{Laboratory}

Full laboratory investigations (blood grouping, CBC, complete liver and kidney functions, blood sugar, bleeding time and clotting time).

Written informed consents were obtained from the cases participating in this study after informing them about the steps of study and possible complications and after approval of Ethical Committee in Faculty of Medicine, Benha University. 


\subsection{Statistical methods}

Information the board and measurable investigation were finished utilizing SPSS vs.25. (IBM, Armonk, New York, United states). Mathematical information was summed up as means and standard deviations. Straight out information was summed up as numbers and rates. Connection investigation was done between age and time before a medical procedure with different boundaries. " $r$ " is the connection coefficient. It goes from -1 to +1 . -1 demonstrates solid negative relationship, +1 shows solid positive connection and 0 shows no connection. Adductor pollicis engine gradings were contrasted as respect consistence with non-intrusive treatment utilizing
Chi-square test. All P esteems were two sided. P esteems under 0.05 were viewed as critical.

\section{Results}

This study was conducted in Orthopedic surgery department, faculty of medicine, Benha university hospitals on ten (10) cases with ulnar nerve injury with proximal forearm injury, The mean time interval before surgery was $4.25 \pm 1.48$ with minimum 2 months and maximum 6 months from injury and healthy median nerve. Mean age of the study population was 31.2 years with stand-ard deviation of 7.66 years. As regard gender, $70.0 \%$ were males while $30.0 \%$ were females Table (1).

Table (1) Demographic characteristics in study population.

\begin{tabular}{|c|c|c|}
\hline \multicolumn{3}{|c|}{ General characteristics } \\
\hline Age (years) & Mean \pm SD & $31.2 \pm 7.66$ \\
\hline Gender & Males $\quad \mathrm{n}(\%)$ & $7(70.0)$ \\
\hline & Females & $3(30.0)$ \\
\hline
\end{tabular}

Majority of study population were manual workers (30.0\%). Other occupations were butcher (10.0\%), driver (10.0\%), farmer $(10.0 \%)$, housewife $(10.0 \%)$, police officer $(10.0 \%)$, student $(10.0 \%)$ and teacher $(10.0 \%)$. The most frequent mechanism of trauma was falling form height $(30.0 \%)$ followed by machine $(20.0 \%)$, road traffic accident $(20.0 \%)$ and sharp knife (20.0\%). The least frequent was by gun shot (10.0\%) Fig (2)

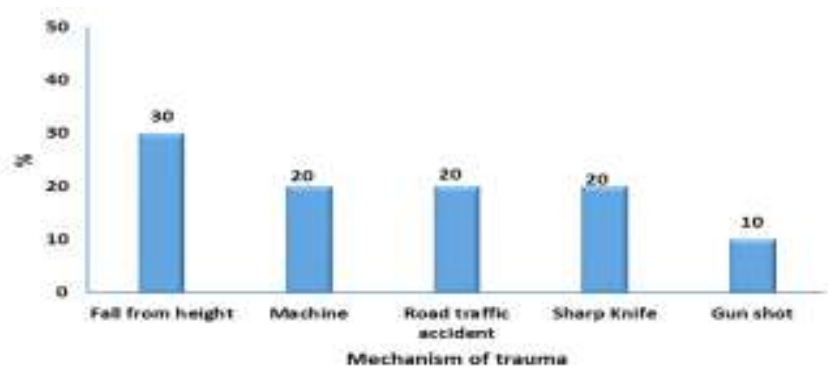

Fig (2) Distribution of different mechanisms of trauma in study population.

\section{Motor outcome after 6 months}

- 3rd palmar interosseus

- $80.0 \%$ showed poor or less grading while $20.0 \%$ showed good grading.

- 2nd palmar interosseus

- $70.0 \%$ showed good grading while $30.0 \%$ showed poor or less grading.

- 1st palmar interosseus

Table (2) 6 months motor grading outcome in study population.
- $50.0 \%$ showed good grading, $30.0 \%$ showed very good grading and only $20.0 \%$ showed poor or less grading.

- Dorsal interossei

- Very good and good gradings were $40.0 \%$ for each and only $20.0 \%$ showed poor or less grading.

- Adductor pollicis: $70.0 \%$ showed very good grading and $30.0 \%$ showed good grading Table (2).

\begin{tabular}{lccc}
\hline & & $\mathbf{N}$ & $\mathbf{\%}$ \\
\hline 3rd palmar interosseus & Poor or less & 8 & 80.0 \\
& Good & 2 & 20.0 \\
& Very good & 0 & 0.0 \\
2nd palmar interosseus & Excellent & 0 & 0.0 \\
& Poor or less & 3 & 30.0 \\
& Good & 7 & 70.0 \\
& Very good & 0 & 0.0 \\
& Excellent & 0 & 0.0 \\
\hline
\end{tabular}




\begin{tabular}{lccc} 
Table (2) Continue & & & \\
\hline 1st palmar interosseus & Poor or less & 2 & 20.0 \\
& Good & 5 & 50.0 \\
& Very good & 3 & 30.0 \\
Dorsal interossei & Excellent & 0 & 0.0 \\
& Poor or less & 2 & 20.0 \\
& Good & 4 & 40.0 \\
Adductor pollicis & Very good & 4 & 40.0 \\
& Excellent & 0 & 0.0 \\
& Poor or less & 0 & 0.0 \\
& Good & 3 & 30.0 \\
& Very good & 7 & 70.0 \\
& Excellent & 0 & 0.0 \\
\hline
\end{tabular}

\section{Functional outcome after 6 months}

- Grip improvement

- $70.0 \%$ showed very good improvement and 30.0\% showed fair improvement.

- Pinch improvement
- $60.0 \%$ showed very good improvement and $40.0 \%$ showed fair improvement.

- Clawing improvement

- Only $20.0 \%$ showed clawing improvement. (Table 3)

Table (3) 6 months functional grading outcome in study population.

\begin{tabular}{lccc}
\hline & & $\mathbf{N}$ & $\mathbf{\%}$ \\
\hline Grip improvement grade & Poor & 0 & 0.0 \\
& Fair & 3 & 30.0 \\
& Very good & 7 & 70.0 \\
Pinch improvement grade & Excellent & 0 & 0.0 \\
& Poor & 0 & 0.0 \\
& Fair & 4 & 40.0 \\
& Very good & 6 & 60.0 \\
Clawing improvement & Excellent & 0 & 0.0 \\
\hline
\end{tabular}

There was a significant negative correlation between age \& adductor pollicis motor grade $(r=-0.853 \& \mathrm{P}$ value $=0.002$ ). There was a significant negative correlation between time before surgery \& adductor pollicis motor grade $(\mathrm{r}=-0.690 \& \mathrm{P}$ value $=0.027)$.

Very good grading was significantly higher in those who showed compliance to physical therapy $(87.5 \%)$ compared to non-complaints $(0.0 \%)$. $\mathrm{P}$ value was 0.016 .

\section{Discussion}

In this investigation the mean old enough was 28.5 \pm 6.86 going from 18-42 years of age which is practically identical with Semaya, [8] as the mean age of the patients in his examination was 24 years (going from 17 to 38 years).

In our investigation the utilitarian misfortune was missing after denervation of pronator quadrates as the pronator teres can redress.

In our investigation follow up to patients went from 6 to 8 months postoperative. Novak and Mackinnon., [9] mean development to their patients postoperative was year and a half. Flores., [10] postoperative subsequent time mean was 20 months (going from 15 to 30 months). In Semaya., [8] study the mean postoperative subsequent period was 22 months (range from 12 to multi month.
The short development in our examination is a disadvantage. Notwithstanding, we will follow our patients later on for longer period to see the drawn out outcomes. Notwithstanding, the outcomes following a half year of follow up are en-couraging. Likewise this may allude to presence of amazing outcomes at different investigations because of long postoperative season of follow up for quite a long time.

In this examination, great engine recuperation (G3 or G4) was seen in patients. The Adductor pollicis longus muscle recuperated (G3) in four patients and (G4) in the other six patients. This brought about great postoperative horizontal squeeze and grasp strength.

In this examination, great engine recuperation was seen in dorsal between ossei muscles in 10 patients to ( 4 patients had G4 and 4 patients had G3 and 2 patients was of helpless outcomes (G2).

As respect to abductor of fingers improvent was seen in first and second palmar between ossei (G3) in 8 patients and 2 patients had helpless result (G2)

The abductor of little finger was noticed poor in all patients $(\mathrm{G} 1 \& \mathrm{G} 2)$ with perseverance of Wartenburg sign in each of the 10 patients.

Novak and Mackinnon., [9] oversaw high ulnar nerve wounds in eight patients by distal nerve move of the AIN to the profound engine part of the ulnar nerve, and no 
tactile nerve move was per-shaped to the tangible part of the ulnar nerve. They demonstrated that all the eight patients had reinnervation of the ulnar nerve inherent hand muscles, with improved postoperative horizontal squeeze and hold strength.

Haase and Chung., [11] oversaw two cases with high ulnar nerve wounds by distal nerve move of the AIN to the profound engine part of the ulnar nerve. They detailed return of capacity to the ul-nar-innervated inherent muscles of the hand in these two cases.

Wang and Zhu., [12] revealed that move of the pronator quadratus part of the AIN to the intermittent part of the middle nerve and the profound part of the ulnar nerve to reestablish the inborn elements of the hand was performed on 10 patients. six patients were followed up for a normal of 68 months. In the most recent development, nor-mal muscle strength (M5) was recovered in three patients, M4 strength in four patients, M3 strength in three patients, and M2 strength in two ptients. No tangible nerve move was acted in these cases.

Our outcomes following AIN to profound engine part of ulnar nerve are near the past examinations.

Our discoveries suggest that better results are altogether associ-ated with more limited Interval before medical procedure and postoperative compli-ance to hand treatment concurring with Secer et al., [4].

In our outcomes we discovered the vast majority of cases had no improvement in mauling so we propose doing ligament move as an auxiliary activity to improve ripping at.

This examination has a few restrictions. The 8 months follow-up probably won't be adequate to assess long haul recuperation.

As far as anyone is concerned, a couple of distributions have utilized similar method of distal neurotization of high ulnar nerve sores utilizing the AIN to reestablish the engine capacity of the ulnar nerve. This investigation clari-fied the signs, the focal points, and inconveniences, and indicated agreeable outcomes contrast and direct nerve fix and ligament move.

\section{Conclusion}

Although the number of patients in our study is small, the proce-dure provides a good option to restore the ulnar nerve functions in cases of high ulnar nerve lesions. The procedure is easy to perform, with minimal donor-site morbidity.

\section{References}

[1] A.Woo, K.Bakri, S.L.Moran. Management of ulnar nerve injuries," J. Hand Surg. Am, Vol.40, PP.173$181,2015$.
[2] N.F.Jones, G. R.Machado. Tendon transfers for radial, median, and ulnar nerve injuries: current surgical techniques," Clin. Plast. Surg, Vol.38, PP.621-642, 2011.

[3] R.M.G.Menorca, T.S.Fussell, J. C. Elfar. Peripheral nerve trauma: mechanisms of injury and recovery," Hand Clin, Vol.29, p. 317, 2013.

[4] H.I.Secer, M.Daneyemez, E.Gonul, Y.Izci. Surgical repair of ulnar nerve lesions caused by gunshot and shrapnel: results in 407 lesions," J. Neurosurg, Vol.107, PP.776-783, 2007.

[5] M.M.Sherif , A.H. Amr. Intrinsic hand muscle reinnervation by median-ulnar end-to-side bridge nerve graft: case report," J. Hand Surg. Am, Vol.35, PP.446-450, 2010.

[6] Q.Li, P Zhang, X.Yin, B. Jiang. Early nerve protection with anterior interosseous nerve in modified end-to-side neurorrhaphy repairs high ulnar nerve injury: a hypothesis of a novel surgical technique," Artif. Cells, Nanomedicine, Biotechnol, Vol.43, PP.103-105, 2015.

[7] J. Isaacs, D.Allen, L. E. Chen, J.Nunley. Reverse end-to-side neurotization," J. Reconstr. Microsurg. Vol. 21, PP.43-48, 2005.

[8] A. Semaya. Reconstruction of high ulnar nerve lesions by distal double neurotization using motor and sensory branches from the median nerve. Egypt. Orthop. J, Vol.50, pp.122, 2015.

[9] C.B.Novak S.E.Mackinnon. Distal anterior interosseous nerve transfer to the deep motor branch of the ulnar nerve for reconstruction of high ulnar nerve injuries," J. Reconstr. Microsurg, Vol.18, PP.459-464,2002.

[10] L.P.Flores.Distal anterior interosseous nerve transfer to the deep ulnar nerve and end-to-side suture of the superficial ulnar nerve to the third common palmar digital nerve for treatment of high ulnar nerve injuries: experience in five cases," Arq. Neuropsiquiatr, Vol.69, PP.519-524, 2011.

[11] S.C.Haase, K.C.Chung. Anterior interosseous nerve transfer to the motor branch of the ulnar nerve for high ulnar nerve injuries," Ann. Plast. Surg, Vol.49, PP.285-290, 2002.

[12] Y.Wang, S.Zhu, B.Zhang. Anatomical study and clinical application of transfer of pronator quadratus branch of anterior interosseous nerve in the repair of thenar branch of median nerve and deep branch of ulnar nerve," Zhongguo xiu fu chong jian wai ke za zhi= Zhongguo xiufu chongjian waike zazhi= Chinese J. reparative Reconstr. Surg, Vol.11, PP.335-337, 1997. 